\title{
ACTIVITY OF TRIAZOLES AND ECHINOCANDINS AGAINST CANDIDA BLOODSTREAM ISOLATES AT PHRAMONGKUTKLAO HOSPITAL, THAILAND
}

\author{
Sudaluck Thunyaharn*, Wichai Santimaleeworagun**, Chananan Khoprasert***, Piyanate Kesakomol**, \\ Montalee Theeraapisakkun ${ }^{* * * *}$,Unchalee Visawapoka****
}

*Faculty of Medical Technology, Nakhon Ratchasima College, Nakhon Ratchasima 30000, Thailand

${ }^{* *}$ Department of Pharmacy, Faculty of Pharmacy, Silpakorn University, Nakorn Pathom 73000, Thailand

*** Department of Microbiology, Phramongkutklao College of Medicine, Bangkok 10400, Thailand

***** Department of Biochemistry, Phramongkutklao College of Medicine, Bangkok 10400, Thailand

\begin{abstract}
Background: Candidemia is a major cause of morbidity and mortality which can be treated using antifungal agents, triazoles and echinocandins.

Objectives: We aimed to determine Candida species and their sensitivities to triazoles (fluconazole, itraconazole, voriconazole, and posaconazole) and echinocandins (caspofungin, micafungin, and anidulafungin) among patients with candidiasis to guide future treatment of patients with candidemia or invasive candidiasis. Methods: All firstly isolated Candida spp. from patients admitted at Phramongkutklao Hospital, Bangkok, Thailand from January 2012 to December 2013 were included in this study. The antifungal susceptibility testing of Candida spp. isolates was assessed based on micro-dilution method.
\end{abstract}

Results: During the 24-month study period, a total of 66 Candida isolates from 66 patients were identified. Of the 66 isolates, 35 (53\%) were C. albicans, 18 (27.3\%) were C. tropicalis, 10 (15.2\%) were C. glabrata and 3 (4.5\%) were $C$. parapsilosis. Fluconazole resistant Candida isolates were found in C. glabrata (100\%), C. albicans (14.3\%), C. tropicalis (22.2\%) and C. parapsilosis (66.7\%). Most Candida spp. isolates were mainly susceptible to echinocandins (>90\%). Notably, $10 \%-20 \%$ of C. glabrata isolates showed resistance to echinocandins.

Conclusion: Fluconazole, an empirical therapy, has been cautiously used due to resistant non-albicans Candida species especially, C. glabrata, C. tropicalis and C. parapsilosis. However, the emerging echinocandins resistant $C$. glabrata isolates need to be closely monitored.

Keywords: Triazoles, Echinocandins, Candida spp., bloodstream infection

J Southeast Asian Med Res 2021: 5 (2):84-90

https://www.jseamed.org

Correspondence to:

Thunyaharn S, Faculty of Medical Technology, Nakhon Ratchasima College, Nakhon Ratchasima 30000, Thailand

E-mail: tanmicro@nmc.ac.th

Received: 25 July 2021

Revised: 09 September 2021

Accepted: 21 September 2021 


\section{Introduction}

At present, the incidence of fungal infections has been increasing due to an increase of immunocompromised patients regarding cancer, organ transplantation and human immunodeficiency virus (HIV). Candida is one of the most common pathogenic fungi causing invasive and noninvasive infections. ${ }^{(1)}$ A meta-analysis of epidemiological studies from Europe from January 2000 to February 2019 showed that invasive candidiasis, particularly candidemia, was associated with high morbidity and mortality rates. ${ }^{(2)}$ A 30-day mortality rate of candidemia among hospitalized patients was approximately $40 \%$. Candidiasis may cause infection in the liver, spleen and brain. Candida infections of the liver or spleen have been major complications among patients with neutropenic cancer. ${ }^{(1)}$

C. albicans is the most common Candida species isolated from clinical specimens. However, prevalence of nonalbicans Candida species has been increasing during the past decade. Among nonalbicans Candida species, prevalence of C. glabrata and C. krusei infections remained unchanged while those of $C$. parapsilosis and C. tropicalis infections have been increasing. ${ }^{(3)}$ Prevalence of $C$. glabrata and C. krusei which harbor intrinsic resistance to triazole antifungul drugs, such as fluconazole ${ }^{(1)}$ have been increasing from $4.9 \%$ in 2001 to $12.3 \%$ in $2010 .{ }^{(4)}$ Thus, changing etiological agents may affect empiric treatment of invasive candidiasis.

From 1999 to 2002, a study at Siriraj Hospital, Thailand, revealed Candida infections totaled 44.6\% while nonalbicans Candida infections totaled $55.4 \%{ }^{(5)}$ The study showed that $C$. albicans, C. tropicalis and $C$. parapsilosis isolates were universally susceptible to fluconazole. However, itraconazole resistant isolates were detected from 16.7 to $19.8 \%$ whereas C. glabrata isolates were predominately resistant to fluconazole. From 2013 to 2015, a multi-center prospective observational study was conducted in seven countries in Asia/ Pacific region; the results showed approximately one fourth of $C$. tropicalis isolates was not susceptible to fluconazole and voriconazole. (6) Moreover, approximately $5 \%$ of $C$. glabrata were nonsusceptible to caspofungin, micafungin and anidulafungin.
As described above, the study of antifungal susceptibility in Thailand was limited, so this study aimed to determine Candida species and activity of triazoles and echinocandins against Candida spp. bloodstream isolates to represent the situation of antifungal options for invasive candidiasis treatment.

\section{Methods}

This study was reviewed and approved by the Ethics Committee of the Medical Department of the Royal Thai Army (approval no. S029b/57).

\section{Fungal strains}

All strains of Candida spp. isolated from blood specimens of patients admitted at Phramongkutklao Hospital, Bangkok, Thailand from January 2012 to December 2013 were obtained. All firstly isolated clinical Candida spp. isolates in each patient were included. Candida isolates were cultured on blood agar at $35^{\circ} \mathrm{C}$ and species of Candida colonies were identified using colony characteristics, germ tube test, and differentiation on CHROMagar accompanied with biochemical tests using a conventional method. In addition, all isolates were processed for matrix assisted laser desorption ionization-time of flight mass spectrometry (MALDI-TOF MS) identification. The pure clinical Candida isolates were kept at $-70^{\circ} \mathrm{C}$ until the antifungal susceptibility test. The regrowth of kept isolates for further testing were cultured on blood agar at $35^{\circ} \mathrm{C}$ from 24 to 48 hours to obtain a pure colony.

\section{Determining antifungal susceptibility}

Antifungal susceptibility testing of Candida spp. isolates was assessed using Sensititre ${ }^{\circledR}$ YeastOne (Thermo Scientific, IL, USA) based on micro-dilution method. The Minimal Inhibition Concentration (MIC) values of tested antifungals obtained from commercial kit test consisted of triazole antifungul drugs (fluconazole, itraconazole, posaconazole, voriconazole) and echinocandins, a class of antifungal drugs (caspofungin, micafungin and anidulafungin). The MIC that inhibited the growth of Candida spp. isolates was determined by changes in the Alamar blue color. For the quality control antifungal test, C. parapsilosis 
ATCC 22019 and C. krusei ATCC 6258 were used as the reference strains according to the Clinical and Laboratory Standards Institute (CLSI) Version M27.

For the MIC breakpoint regarding susceptibility interpretation, we used the epidemiologic cut-off values (ECVs) and clinical breakpoints based on the CLSI M59 $2^{\text {nd }}$ edition and CLSI M60 version, respectively. In the case of lacking breakpoints based on CLSI, we used the clinical breakpoints and ECVs based on the European Committee on Antimicrobial Susceptibility Testing (EUCAST) 2019 guidelines.

\section{Results}

During a 24-month study period, 66 Candida specimens were clinically isolated. Of these, 35 (53\%) were C. albicans. Other nonalbicans Candida isolates comprised the following: $C$. tropicalis (18, 27.3\%), C. glabrata $(10,15.2 \%)$ and $C$. parapsilosis (3, 4.5\%). Generally, C. glabrata isolates were mostly resistant to triazoles such as fluconazole (susceptible rate $0 \%$ ) and posaconazole (susceptible rate $40 \%$ ), except itraconazole (susceptible rate $90 \%$ ) and voriconazole (susceptible rate 90\%). C. tropicalis was resistant to fluconazole and itraconazole with resistant rates of 22.2 and $33.3 \%$, respectively. However, Candida spp. isolates were mainly susceptible to echinocandins with susceptible rates from 80 to $100 \%$ (Table $\mathbf{1}$ ).

Not all C. albicans isolates were susceptible to fluconazole. The results of MIC range, MIC50 and MIC90 for fluconazole against C. albicans were as follow: 0.12 to $>256 \mu \mathrm{g} / \mathrm{mL}, 1 \mu \mathrm{g} / \mathrm{mL}$ and $128 \mu \mathrm{g} / \mathrm{mL}$, respectively. Moreover, MIC range, MIC50 and MIC90 for fluconazole against C. tropicalis were: 0.12 to $8 \mu \mathrm{g} / \mathrm{mL}, 1 \mu \mathrm{g} / \mathrm{mL}$ and $4 \mu \mathrm{g} / \mathrm{mL}$, respectively. Table 1 shows the ranges of antifungal MIC values against each Candida spp. isolate.

\section{Discussion}

Studies of Candida bloodstream isolates among patients admitted in tertiary care hospitals have been reported in Thailand. From 1999 to 2002, the prevalence of $C$. albicans comprised $44.6 \%$ while nonalbicans Candida spp. isolates accounted for 55.4\% ${ }^{(5)}$ Additionally, from 2004 to 2009, C. albicans and nonalbicans Candida accounted for 40.3 and $59.7 \%$, respectively. ${ }^{(7)}$ Prevalence of non-albicans Candida species over $C$. albicans was also similar to recent reports among countries in the Asia-Pacific region ${ }^{(6,8)}$

This study reported on the species distribution of Candida bloodstream isolates among patients admitted in Phramongkutklao Hospital and their antifungal susceptibilities from 2012 to 2013. During the study period, 66 Candida isolates were identified. Our study showed that $C$. albicans was the most predominant species followed by $C$. tropicalis, C. glabrata and $C$. parapsilosis. In contrast to a study of Candida species isolated from patient blood samples at Siriraj Hospital, Bangkok, Thailand from January 2016 to December 2017, C. tropicalis was the most predominant, followed by $C$. albicans, $C$. glabrata and C. parapsilosis. Species distribution of Candida spp. directly affected the optimal candidemia treatment. Thus, the close monitoring and surveillance in each hospital setting was important to appropriately design institutional guidelines for empiric treatment of invasive Candida infection.

According to 2016 clinical guidelines to manage candidiasis by the Infectious Diseases Society of America, either nonneutropenic or neutropenic patients, receiving a diagnosis of invasive candidiasis, are strongly recommended to undergo echinocandins treatment as first line empirical therapy. However, fluconazole should be used as an alternative drug in the case of non-critically ill or nonsuspected infection with fluconazoleresistant Candida spp., especially C. krusei or C. glabrata infections. $^{(10)}$ Our study showed that C. glabrata, accounted for $15 \%$ of all Candida bloodstream isolates, and was universally resistant to fluconazole. Moreover, fluconazoleresistant Candida spp. were detected in C. albicans $(14.3 \%)$ and $C$. tropicalis $(22.2 \%)$ isolates. Additionally, a recent study using Candida spp. isolated from blood samples collected from 2016 to 2017 reported that fluconazole resistance was significantly increased in C. tropicalis $(37.8 \%)$. (11) Thus, treatment using fluconazole was concerned for fluconazole-resistant C. tropicalis. 


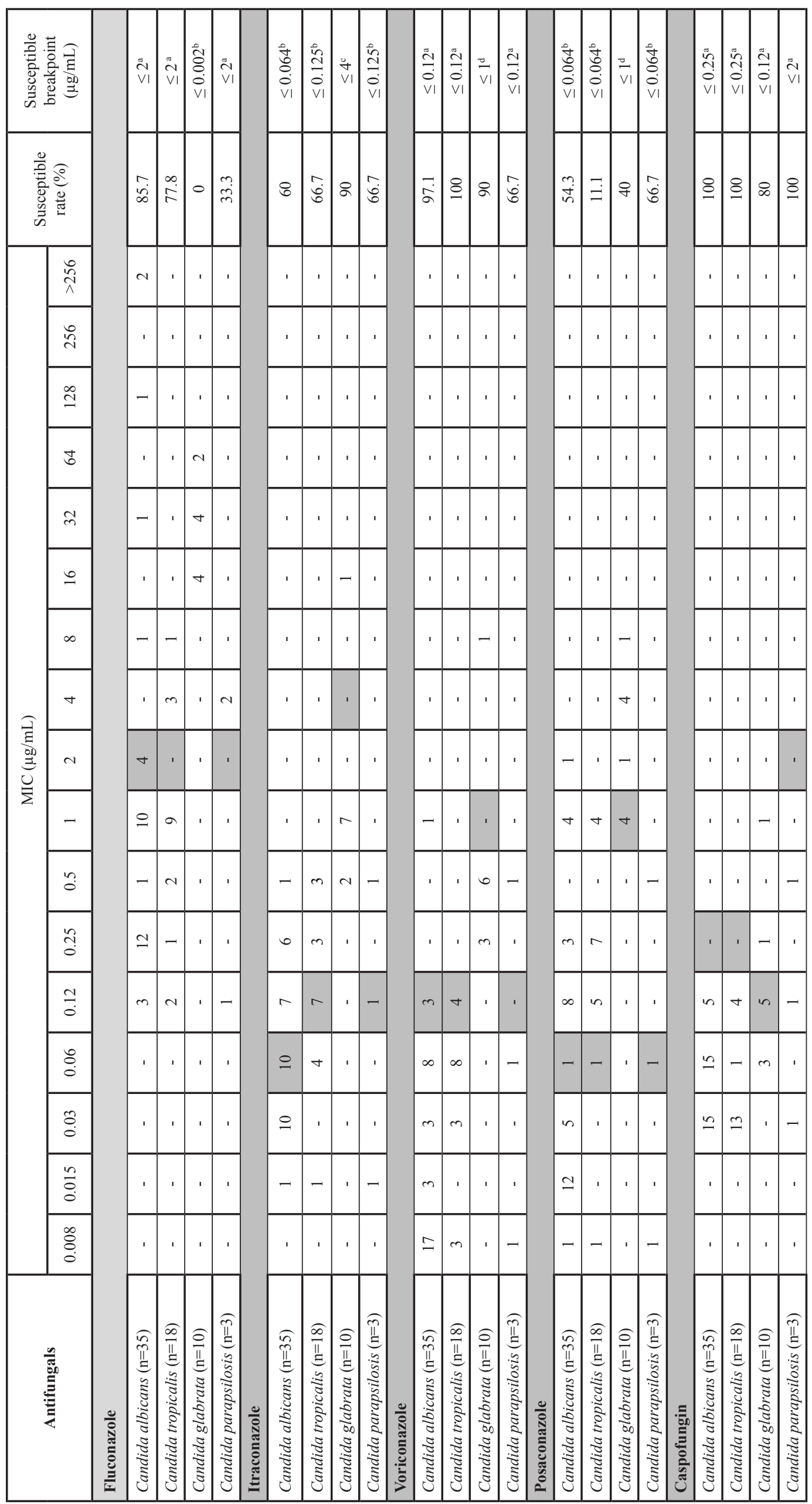




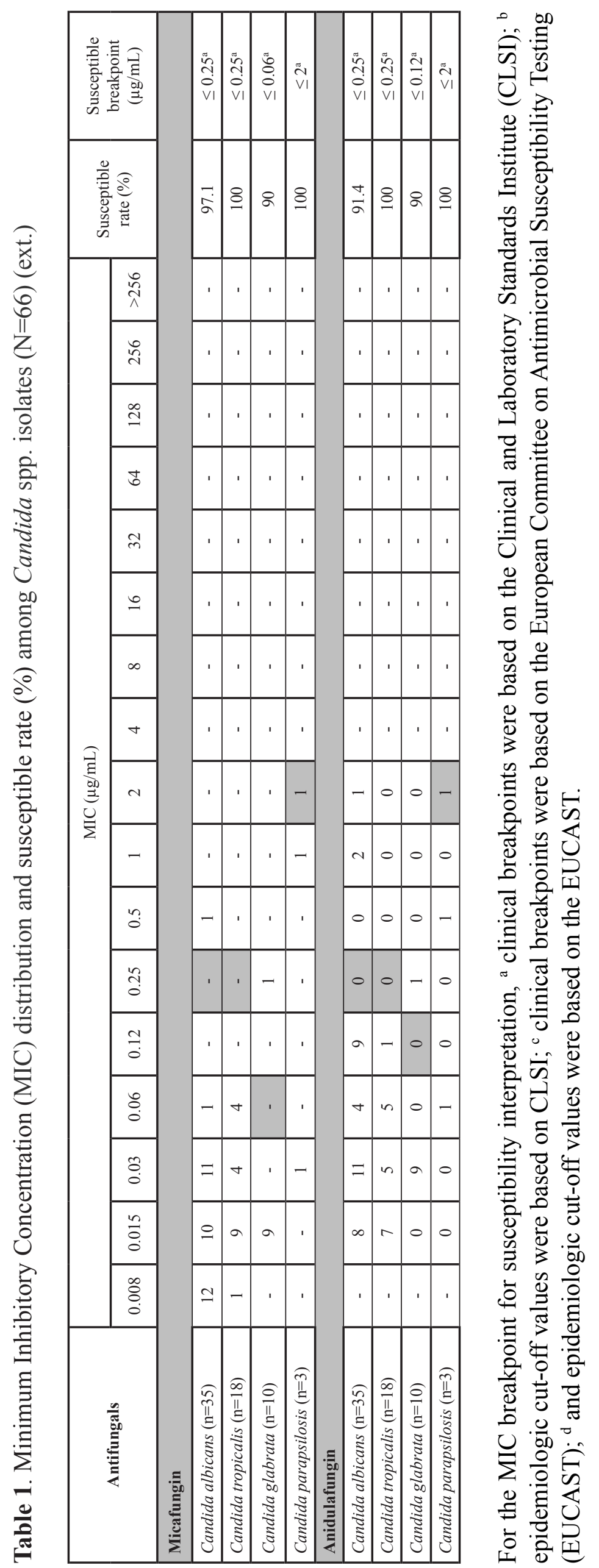


Consequently, this study showed over $80 \%$ of Candida spp. isolates remained susceptible to caspofungin, micafungin, and anidulafungin. As a result, echinocandins seemed to be the preferable choice for Candida bloodstream infection. However, $20 \%$ of $C$. glabrata isolates were resistant to caspofungin compared with 5\% reported from countries in the Asia/Pacific region. ${ }^{(6)}$ Thus, further studies need to closely monitor the echinocandins resistant Candida species when echinocandins has been used as empirical therapy.

The oretically, MICs to echinocandins against C. parapsilosis usually tend to be higher due to intrinsic resistance that should lead to less successful treatment by echinocandins. $C$. parapsilosis isolates still comprised echinocandins susceptible strains. In Spain, among 200 episodes of $C$. parapsilosis bloodstream infection, initial use of an echinocandin-based regimen had no impact on clinical failure. ${ }^{(12)}$ Thus, echinocandins remains a preferably empiric choice regarding C. parapsilosis infection as one of the etiologic candidemia.

In this study, results were obtained from in vitro assay of antifungal activity against identified Candida spp. Practically, for each healthcare setting, selection of antifungal therapy has to be based on Candida species identification, antifungal susceptibility pattern, patients' severity of illness and underlying diseases, and co-administered medications including certain drugs prescribed in some patients' conditions.

\section{Conclusion}

Prevalence of Candida bloodstream species and the role of echinocandins as empirical therapy were investigated. Due to drug resistance of nonalbicans Candida spp., especially, C. glabrata, C. tropicalis and C. parapsilosis, fluconazole, an alternative choice for invasive candidiasis, should be used cautiously. Echinocandins remains the preferable choice for candidemia; however, the increase of echinocandins resistant $C$. glabrata isolates needs to be closely monitored.

\section{Acknowledgments}

The authors are thankful to Phramongkutklao College of Medicine for financial support and providing the required facilities for this research.

\section{References}

1. Enoch DA, Yang H, Aliyu SH, Micallef C. The changing epidemiology of invasive fungal infections. Methods Mol Biol 2017; 1508: 17-65.

2. Koehler P, Stecher M, Cornely OA, Koehler D, Vehreschild M, Bohlius J, et al. Morbidity and mortality of candidaemia in Europe: an epidemiologic meta-analysis. Clin Microbiol Infect 2019; 10: 1200-12.

3. Guinea J. Global trends in the distribution of Candida species causing candidemia. Clin Microbiol Infect 2014; 20 (Suppl 6): 5-10.

4. Alexander BD, Johnson MD, Pfeiffer CD, Jimenez-Ortigosa C, Catania J, Booker R, et al. Increasing echinocandin resistance in Candida glabrata: clinical failure correlates with presence of FKS mutations and elevated minimum inhibitory concentrations. Clin Infect Dis 2013; 56: 1724-32.

5. Foongladda S, Sakulmaiwatana P, Petlum P, Vanprapar N. Candida species, genotypes and antifungal susceptibility of Candida isolates from blood samples of patients at the largest tertiary care hospital in Thailand during 1999-2002. J Med Assoc Thai 2004; 87: 92-9.

6. Tan TY, Hsu LY, Alejandria MM, Chaiwarith $\mathrm{R}$, Chinniah T, Chayakulkeeree $\mathrm{M}$, et al. Antifungal susceptibility of invasive Candida bloodstream isolates from the Asia-Pacific region. Med Mycol 2016; 54: 471-7.

7. Jutiamornlerd N, Chusri S, Siripaitoon P. Epidemiology of candidemia in Songklanagarind Hospital. J Med Assoc Thai 2011; 94: 927-32.

8. Tan BH, Chakrabarti A, Li RY, Patel AK, Watcharananan SP, Liu Z, et al. Incidence and species distribution of candidaemia in Asia: a laboratory-based surveillance study. Clin Microbiol Infect 2015; 21: 946-53.

9. Chaiwarith R, Ounbang P, Khamwan C, Nuntachit N, Sirisanthana T, Supparatpinyo K. Epidemiology of adult candidemia at Chiang Mai University Hospital. Southeast Asian J Trop Med Public Health 2011; 42: 1505-14.

10. Pappas PG, Kauffman CA, Andes DR, Clancy CJ, Marr KA, Ostrosky-Zeichner L, et al. Clinical practice guideline for the management 
of candidiasis: 2016 Update by the Infectious Diseases Society of America. Clin Infect Dis 2016; 62: e1-50.

11. Ngamchokwathana C, Chongtrakool P, Waesamaae A, Chayakulkeeree M. Risk factors and outcomes of non-albicans Candida bloodstream infection in patients with candidemia at Siriraj Hospital-Thailand's largest national tertiary referral hospital. J Fungi (Basel) 2021; 7: 269.

12. Fernandez-Ruiz M, Aguado JM, Almirante B, Lora-Pablos D, Padilla B, Puig-Asensio M, et al. Initial use of echinocandins does not negatively influence outcome in Candida parapsilosis bloodstream infection: a propensity score analysis. Clin Infect Dis 2014; 58: 1413-21. 